\title{
Nature Biotechnology's academic spinouts of 2019
}

\author{
Our annual survey highlights startups tackling intractable viruses with new vaccine design, engineering a reliable \\ source of platelets, universalizing cell therapies, improving cancer screening, developing RNA-editing platforms and \\ targeting protein-RNA interactions. Michael Eisenstein, Ken Garber, Caroline Seydel and Laura DeFrancesco report.
}

T he universe of life science ventures flourished last year. Some of the largest rounds of venture capital ever seen went into 2019 biotech startups. Several of these are profiled here, as are companies that are working with less capital but still breaking new ground (Table 1).

As in previous years of our survey, we have focused on R\&D-intensive startups spun out from academic institutions. These were first identified as having raised a series A financing in 2019. Our editors then assessed publicly available information about each firm's research to select those that appear below.

(Some firms were selected but not included because they were still in 'stealth mode' or declined to be interviewed.)

What follows are the stories behind the selected ventures and their offerings. Although our survey is by no means exhaustive, we believe these companies represent some of the best (and most handsomely financed) science that was commercialized from academia in 2019.

\section{Sana Biotechnology: universalizing cell therapy}

Until recently, one could be forgiven for thinking that gene therapy might well remain 'the medicine of the future' - always just over the horizon, but still short of reaching the market. Today, that promise is finally a reality, with regulatory approvals for cell therapies, such as chimeric antigen receptor (CAR) T cells developed for cancer immunotherapy by Kite Pharma (now part of Gilead Sciences) and Novartis, as well as Spark Therapeutics' voretigene neparvovec

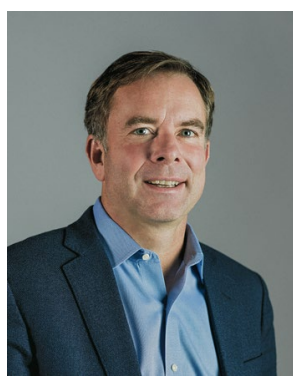

(Luxturna) gene therapy for retinal dystrophy.

At the same time, these breakthroughs are specific for a narrow range of diseases and tissues, and cell and gene therapy remains some way short of Steve Harr, president and CEO, Sana Biotechnology. system that enables therapeutic delivery to any desired tissue or cell. This is the challenge that Sana Biotechnology aims to tackle. According to president and CEO Steve Harr, Sana's goal is no less than to develop strategies that enable the repair or replacement of any cell in the body. "This isn't about trying to build a single tool," says Harr. "We're building a toolbox." To start, this will entail a two-pronged approach, with the company developing 'universal' stem cells that can be employed for off-the-shelf regenerative medicine applications without fear of immune rejection, as well as a novel approach to the targeted genetic modification of cells and tissues in vivo.

The company has assembled a host of cell and gene therapy veterans to achieve these goals. Among the first to sign on was Richard Mulligan, one of gene therapy's founding fathers. Mulligan saw Sana's work as a unique opportunity. "After 30 years of doing this type of thing, the one thing I thought was a real failure was my inability to usher this raw scientific technology down a clinical pathway," he says. In particular, he was struck by the company's focus on developing broad platform technologies rather than solutions to more narrowly defined problems.

Sana drew a reported $\$ 219$ million in funding in 2019 from backers including ARCH Venture Partners, Flagship Pioneering and F-Prime Ventures, and is already valued at more than $\$ 1$ billion. In addition to Mulligan, who serves as vice president of the company's board of directors, Sana's leadership is stacked with cell and gene therapy experts from Juno Therapeutics, one of the first companies to pursue clinical development of CAR T-cells. This includes Harr, former CFO and head of corporate development, and Sana's head of R\&D, Sunil Agarwal, who served the same role at Juno. Academic research also plays a foundational role in the company's technology portfolio, most notably in the realm of universal stem cell production. The company's cell engineering strategies draw on work by a Sana cofounder and former CSO, the Harvard Stem Cell Institute researcher Chad Cowan, and Sonja Schrepfer of the University of California, San Francisco, now head of Hypo-Immune Platform at Sana.

These programs are focused on induced

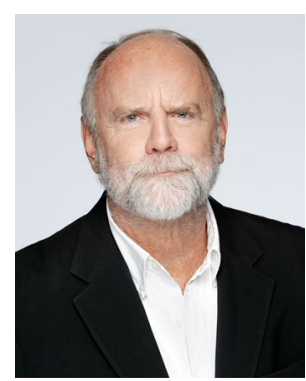

Richard Mulligan, executive vice chairman, Sana Biotechnology. genetically reprogrammed to enter a state that closely resembles embryonic stem cells, but without the legal and ethical burdens of those cells. These cells can then be genetically modified as desired and cultivated under conditions that promote differentiation into a host of mature cell types that are potentially suitable for transplantation. But unless a patient receives treatment with autologous iPSCs derived from their own tissues - a costly and labor-intensive approach to therapy - host rejection is almost certain. "Basically, anywhere that's not immune-privileged, you will have a very avid immune system ready to destroy your very expensive cell therapy," explains Tracy Grikscheit, a pediatric surgeon specializing in regenerative medicine at Children's Hospital Los Angeles. "There are even some reports that autologous cells may run a risk." As a consequence, early-stage clinical development of iPSC-based therapies has focused on indications in tissues known to have less stringent immune surveillance than most others, such as macular degeneration in the eye or Parkinson's disease in the brain.

The immune 'cloaking' strategy employed by Schrepfer's group entails the use of CRISPR-mediated genome editing to delete genes encoding components of the major histocompatibility complex (MHC) while boosting production of a protein called CD47, the so-called 'don't eat me' signal that protects cells from consumption by macrophages and dendritic cells. This confers two-pronged protection for these cells, according to Harr. "Things we do to modify the adaptive immune system tend to make things worse with the innate immunity - she figured out a way to modify cells to cloak them from both arms," he says. The Cowan group's approach went even further, making additional CRISPR modifications to induce expression of programmed cell death 


\begin{tabular}{|c|c|c|c|}
\hline Company & Focus area & $\begin{array}{l}\text { Funding } \\
\text { (\$ millions) }\end{array}$ & Scientific founder \\
\hline Elicio Therapeutics & Amphiphile technology & 46 & D. Irvine (MIT) \\
\hline $\begin{array}{l}\text { Thrive Earlier } \\
\text { Detection }\end{array}$ & CancerSEEK diagnostic & 110 & B. Vogelstein (JHU) \\
\hline Icosavax & $\begin{array}{l}\text { Virus-like particle } \\
\text { vaccine }\end{array}$ & 51 & N. King, D. Baker (UW) \\
\hline Platelet BioGenesis & Platelets & 26 & $\begin{array}{l}\text { J. Thon (Harvard), L. Mazutis, } \\
\text { J. Italiano (Harvard) }\end{array}$ \\
\hline Sana Biotechnology & Cell and gene therapy & 219 & $\begin{array}{l}\text { D. Baker (UW), C. Cowan (HMS), } \\
\text { C. Murry (UW), S. Schrepfer } \\
\text { (UCSF), R. Mulligan (HMS) }\end{array}$ \\
\hline Twentyeight-seven & $\begin{array}{l}\text { Modulation of RNA } \\
\text { modifying enzymes }\end{array}$ & 82.75 & $\begin{array}{l}\text { G. Daley, R, Gregory, P. Sliz, } \\
\text { F. Slack (Harvard) }\end{array}$ \\
\hline Shape Therapeutics & RNA editing in vivo & 35.5 & P. Mali (UCSD) \\
\hline Korro Bio & RNA editing in vivo & 4 & J. Rosenthal (MBL) \\
\hline
\end{tabular}

MIT, Massachusetts Institute of Technology; JHU, Johns Hopkins University; UW, University of Washington; HMS, Harvard Medical School; UCSF, University of California, San Francisco; UCSD, University of California, San Diego; MBL, Marine Biological Laboratory.

ligand 1 (PD-L1), an immunosuppressive protein that fends off autoimmunity, as well as another protein called human leukocyte antigen G (HLA-G). HLA-G protects fetuses from rejection by the mother's immune system during gestation, and conferred similar benefits when presented on the surface of modified iPSCs. "It's a beautiful combination of removing signals to come into battle and also putting up peace signs," says Grikscheit.

However, these approaches have only been tested in mice and cultured cells to date, and many questions remain. Grikscheit notes that the full range of determinants of immune tolerance and rejection remain poorly understood and that it may be difficult to win such an arms race through further modification. "The more you reprogram cells, the more likely they are to not follow the program," she says. In the end, only testing in more sophisticated animal models - and eventually humans - will provide the answers. There may also be unintended consequences from having transplanted cells that are essentially unaccountable to immune oversight, especially if they become tumorigenic or infected. "For any program that moves forward here, it would be advantageous for us to have a way to control the outcome of the cell, such as a cellular 'suicide switch", says Harr.

But the rewards from a successful cloaking strategy could be tremendous. With access to a manufacturing pipeline of one-size-fits-all stem cells, genetically modified donor tissues could become broadly accessible for a host of medical conditions. "We're focused on cells that would have major impact on diseases that a lot of people get," says Mulligan. "These aren't orphan diseases - we're talking about heart disease, or liver disease, or diabetes."

Cell therapy isn't the only arrow in Sana's quiver, however. The company is also looking to commercialize a novel strategy developed by another Flagship-backed startup, Cobalt Biomedicine, which Sana has since acquired. This entails the use of 'fusogens' - retroviral or lentiviral vectors or particles incorporated into a lipid bilayer that can facilitate targeted delivery and uptake of a gene encoding a protein of interest with a regulatory element (for example, a liver-cell-specific promoter) targeting a specified cell type.

Mulligan sees this fusogen technique as a possible alternative to gene therapy strategies that rely on the transplantation of genetically modified hematopoietic stem cells. At present, this requires complicated extraction and cultivation procedures to prepare the bone-marrow-derived donor cells, as well as grueling treatments with chemotherapy or radiation to kill off existing marrow stem cells and prepare recipients for transplant. "We've probably done more mouse bone marrow transplants than anyone in the world, and it would be really spectacular to throw that away and be able to directly just shoot in some virus," says Mulligan.

The fusogen platform and the immune-cloaked stem cell platforms are still years from clinical testing, but Mulligan is excited by Sana's willingness to think into the future. Indeed, he heads up a 'skunkworks' division of the company known as SanaX, which focuses on technologies that might become application-ready on a five- to ten-year timeframe. But he also credits Harr and the rest of the executive team for their focus on practical implementation. Says Mulligan, "We don't have the facilities yet, but we have a first-rate group poised to make sure that when we need to manufacture lentivirus or hypo-immune cells, we'll have them." Over the next couple of years, Harr's goal is to refine and prove out the Sana platform in a preclinical setting and, ideally, to obtain some robust data from animal models to justify preparations for human trials - although he acknowledges the risks that come with the development of novel cell and gene therapy technologies. "It's not going to be a linear path to success," he says. "But if we get it right, it will really matter."

\section{Thrive Earlier Detection: finding cancer} early and often

Imagine sitting in your doctor's office for your annual exam. The doctor sends you for a routine blood draw, with orders to check cholesterol, iron levels and ... cancer biomarkers? Bert Vogelstein, cofounder of Thrive Earlier Detect, envisions just such a scenario. Thrive's blood test, CancerSEEK, detects telltale signatures of eight cancer types, including five for which screening tests are non-existent.

Ever since genetic mutations were linked to cancer, researchers have sought to exploit those associations. Vogelstein, a professor of oncology at Johns Hopkins University, pioneered the mutation model of cancer back in the early 1990s. "Most people at the time - and still now - are thinking, if you know the mutations, you can develop new therapies," he says. "My feeling was a bit different."

Instead, Vogelstein advocates a strategy of early detection, using mutations as biomarkers to identify cancers before they

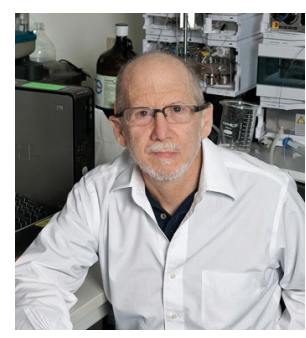

Burt Vogelstein, cofounder, Thrive Earlier Detection (courtesy Thrive Earlier Detection). metastasize, while they can still be cured by surgery. The quest to develop a simple, non-invasive test to flag cancer cells has spurred a hotbed of innovation. So-called 'liquid biopsies' test bodily fluids, such as blood or urine, for signals of cancer, but so far these 
tests have been most useful for detecting a cancer's response to therapy or for early warning of recurrence.

With CancerSEEK, the goal is to reveal cancer in asymptomatic individuals by analyzing circulating DNA and protein biomarkers in blood. Using a machine-learning algorithm, the inventors trained software to evaluate the combination of DNA mutations and a panel of protein biomarkers to deduce where cancer might be originating.

Cancer cells, like healthy cells, release free DNA into the bloodstream. Thrive's test hinges, in part, on the ability to detect oncogenic mutations in that circulating tumor DNA. But in early-stage cancer, there may be just one mutant DNA molecule for every 10,000 sequenced. Although massively parallel sequencing instruments provide the high throughput needed to find that one molecule, their error rates are too high to reliably confirm the mutation.

"If you want to detect a cancer through that approach, you have to be able to distinguish between low-frequency mutations derived from cancer and the background artifacts of the sequencing process itself," says Isaac Kinde, cofounder of Thrive and head of research and innovation for the company.

Kinde developed a method that can do just that. "The Safe Sequencing System reduces the error rate of sequencing to make low-frequency mutation detection more reliable," he explains. The system involves tagging individual template DNA molecules at the start of the reaction with unique identifiers, then amplifying them. If there's a mutation in the original source DNA, that mutation will show up in every molecule containing that same identifier. Mutations that are introduced later will only be seen in a smaller fraction of the DNA strands bearing that tag.

Once they gained the ability to find the needle in the haystack, the researchers needed to decide how many different needles to search

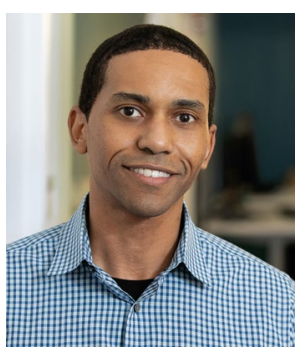
for. Check too few mutations, and many tumors will slip by undetected. Too many, and you boost the chance of false positives - as well as ratcheting up the cost.

In the 2018

Isaac Kinde, cofounder and head of research and innovation, Thrive Earlier Detection (courtesy Thrive Earlier Detection).
Hopkins analyzed the test's ability to detect eight different cancer types - ovary, liver, stomach, pancreas, esophagus, colorectum, lung or breast - in patients who had been diagnosed with stage 1 to stage 3 cancer. Based on that work, they capped the number of genes tested at 16, to hit the sweet spot between increasing sensitivity and decreasing specificity.

Unlike other liquid biopsies in development, CancerSEEK combines mutation analysis with protein biomarkers to increase specificity and identify the tissue where the tumor resides. The test evaluates 1,933 distinct genomic positions and 8 protein biomarkers, including CA-125, carcinoembryonic antigen (CEA), myeloperoxidase and prolactin.

"What I have been most impressed with in their approach is the idea that there's no one test fits all," comments Pierre Massion at Vanderbilt Ingram Cancer Center in Nashville, Tennessee. "I think it's a good idea to combine approaches to reach the same goal." He cautions, however, that protein biomarkers are only informative if they're tested in the right context. "If you develop a biomarker in the context of advanced disease and try to test the biomarker in early disease, you're more likely to fail," he says.

Indeed, in that first study, which included 1,005 patients, CancerSEEK successfully detected only $43 \%$ of stage 1 cancers, compared with $76 \%$ of stage 2 and $78 \%$ of stage 3 cancers. "It's not going to be a slam dunk, and it's never a slam dunk really, but the field is going in the right direction," says Massion. "It's a very exciting time."

The field is indeed abuzz with different companies coming at the early detection problem from different angles. Guardant Health, which already offers a blood test to inform treatment selection for patients with advanced cancer, is enrolling patients in a trial for an early-detection blood test targeting colorectal cancer. In March of this year, startup Grail published results from a trial of 6,700 people, in which their blood test successfully detected 50 different types of cancer with an overall sensitivity of $44 \%$ for stage 1-3 cancers. Grail's test detects circulating tumor DNA on the basis of unique methylation patterns that distinguish it from the DNA of healthy cells.

Thrive Earlier Detection launched in May 2019 with an astonishing \$110 million in series A funding, led by Third Rock Ventures. "The people at Third Rock read the paper and were very excited about it," recalls Vogelstein. "They thought it was a golden opportunity to make a difference for people."

"It immediately got my attention," recalled Steve Kafka, who was a venture partner at Third Rock at the time. "I just really jumped in, because first, the mission itself is so compelling, but also we finally have a scientific approach that's going to be clinically and commercially viable." Kafka, who served as president of Foundation Medicine from 2013 to 2018, signed on as the founding CEO of Thrive, a position he held until August of 2019.

Kafka currently serves as the chairman of Thrive's board of directors and as a managing partner with Section 32, another of the company's investors.

The company recently completed a large, prospective interventional study to see how the test performs in a more real-world context. The DETECT-A study, conducted in conjunction with Geisinger Health, enrolled 10,000 women ages 65-75 with no history of cancer. Results are expected to be published soon.

"The next big event will be a registrational trial with the FDA," says David Daly, Thrive's CEO since August 2019. Although the details have yet to be made final, the trial will include both men and women, and a much broader demographic more representative of the general population.

One critical issue in the DETECT-A results will be the false-positive rate. The test sometimes returns false positives due to the protein biomarkers, which may signal other health conditions unrelated to cancer. Whereas initial testing compared patients with cancer to healthy controls, routine screening will mean testing a wider array of people, some of whom may have other medical conditions.

"There are protein elevations that can be dramatic that are not specific to cancer," notes Kinde. Myeloperoxidase, for example, is associated with inflammation from a variety of diseases. "We analyze the protein biomarkers in a way that achieves high specificity, but it's not perfect," he says.

But Kinde also points out that some false positives could be in fact true, but too small to be verified by conventional means. Because CancerSEEK detects cancer arising from almost anywhere in the body, a positive result may require some searching to find the source cancer. The test is able to narrow the tissue of origin to two possible organs in most cases, but the accuracy varies on the basis of the tumor type, so follow-up imaging is needed.

"If you find a cancer, but on a subsequent imaging study can't identify where the cancer is, that appears to be a false positive," Kinde says, adding "in reality, we could just be detecting disease at an early enough stage that conventional follow-up techniques don't have the sensitivity that matches CancerSEEK. But how commonly this 
scenario occurs will not be clear until we have additional longitudinal patient data"

Another hurdle Thrive is preparing to leap is the challenge of inserting the test into the typical workflow of a busy primary care physician. To encourage doctors to add CancerSEEK to their repertoire, Thrive is developing an integrated service component that will guide patients and providers through the process of understanding and following up on - either a positive or a negative test result. The test won't help anybody, they reason, unless doctors and patients understand what the results mean.

"CancerSEEK is really designed with that integration to routine practice in mind," says Kafka. "It's easy for the physician to use, it's affordable for the payer, and it has the robustness of the result that we believe regulators and ultimately physicians can rely on." He expects that the test's specificity and its cost-benefit profile will appeal to payers. "It isn't easy to get Medicare or the private insurers to do something novel," he says.

"I don't think they look at themselves just as a company that's going to sell a test," Vogelstein says of Thrive. "They look at themselves as a cancer healthcare company." A key message for patients to understand, he explains, is that the blood test should supplement, not replace, currently recommended screening tests. "How do you avoid the patient saying 'I had the blood test, I don't need my mammography," he says.

"You don't want that to happen."

"The overarching purpose of startup companies that arise out of academia is to make interesting discoveries into useful products for the public," Vogelstein states. "That's why it's so important to have industry working with academics, and I think that Thrive is an excellent example of how that can be done in a pretty seamless fashion."

Icosavax: building vaccines de novo In this age of the coronavirus, all eyes are on vaccine developers. Various designs have been employed for virus vaccines, from the earliest vaccines of inactivated virus to the latest genome-based versions. Nonetheless, some viruses have remained intractable no matter the design. One such virus is respiratory syncytial virus (RSV), which causes a pneumonia that kills 160,00 people annually. But now researchers at the University of Washington's Institute for Protein Design (IPD) have created a self-assembling nanoparticle that, in animal models, induces neutralizing antibodies against RSV more than ten times as well as seen with prior designs. This fully synthetic platform, which has been the foundation for the Seattle-based start-up Icosavax, holds

out hope of cracking some of the toughest vaccine problems.

"Some of the coolest, most amazing proteins are those that self-assemble," says Neil King of the



Neil King, cofounder and chair of scientific advisory board, Icosavax (courtesy Institute of Protein Design). IPD, a coauthor on their seminal Cell paper, who now chairs Icosavax's scientific advisory board. "If we can design new self-assembling proteins, that will be a key step toward designing novel molecular machines that are customized for specific applications." A computer program called

Rosetta, created in David Baker's lab at the IPD in the late 1990s and since then developed by a worldwide consortium of contributors, enables researchers to design synthetic proteins by first envisioning the three-dimensional structure that they want to create. Early versions of Rosetta predicted the structures of proteins on the basis of their amino acid sequence, but throughout 20 years of continuous development, its capabilities have expanded considerably. By 2012, Rosetta could reliably go the other direction, predicting the amino acid sequence that will fold into a desired conformation, although loops and side chains in large proteins still present challenges. "The magic, or the core piece of the technology, is that we can do this with atomic-level accuracy," says King.

That precise control allowed King to take the technology even further, designing pairs of peptides that would arrange themselves into a complex quaternary structure. "We use the software to model three-dimensional arrangements of protein subunits," he said, "then we design new protein-protein interfaces between those subunits that drive assembly to the target structure."

"Once we had the ability to design self-assembling nanomaterials, we wanted immediately to apply them to improved public health," says King. "The technology was very well suited to vaccines."

Icosavax is setting its sights on RSV, which is particularly deadly for young children in the developing world who lack access to interventions such as mechanical ventilators. An early RSV vaccine trial ended in tragedy, setting the field back for decades. A vaccine made from formalin-killed virus not only failed to protect babies from infection, but worsened the outcomes of those who got infected. Among children receiving the vaccine, $80 \%$ of those who became ill required hospitalization, compared with $5 \%$ of the control group, and tragically, two vaccinated children died. The reason for this outcome remained a mystery until 2009, researchers reported that the problem was due to the production of low-affinity antibodies that failed to neutralize RSV but increased the severity of the disease by depositing immune complexes in the lungs.

In the meantime, the debacle firmly ruled out a killed-virus vaccine. Two other possible strategies remained: live attenuated virus or a recombinant vaccine created with protein subunits isolated from the viral particle. Much effort has gone into developing a live attenuated virus, but so far researchers haven't been able to stimulate a durable immune response.

The subunit approach, of course, has its own drawbacks. The RSV protein that elicits an immune reaction, the $\mathrm{F}$ protein, exists in two configurations. The active form, called the pre-fusion conformation, is unstable and difficult to purify. It prefers to shift into the less immunogenic post-fusion conformation, but vaccines using the stable form of the antigen haven't been effective.

Jason McLellan developed the F protein antigen that Icosavax licensed for use on their synthetic particles when he was a postdoc at the Vaccine Research Center in the US National Institute of Allergy and Infectious Diseases. He and his colleagues trapped the pre-fusion protein with antibodies to prevent it from flipping into the post-fusion conformation. With the protein held in place this way, they performed X-ray crystallography to scrutinize its atomic structure, and in doing so they uncovered a way to modify and stabilize it. By adding a disulfide bond and filling two hydrophobic cavities, they successfully fixed the protein in the active state. "We call it 'DS-Cav1' because of the disulfide bond (DS) and cavity-filling (Cav1) mutations," said McLellan, who is now at the University of Texas, Austin. "When we injected this, we got antibody titers that were tenfold higher than the post-fusion form."

Various companies have licensed the antigen for vaccine development. Icosavax's unique approach combines the stabilized antigen with a technique designed at IPD for creating self-assembling nanoparticles. To optimize the antibody response, they attach the antigen to a virus-like particle (VLP), a shell lacking genetic material. The VLP mimics the symmetric and repetitive structure of the viral capsid that serves as 
a red flag attracting the full brunt of the immune system's attack.

"The body reacts very differently if it sees a virus than if it sees a simple soluble protein," says

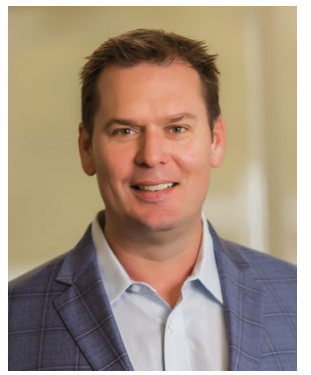

Adam Simpson,

CEO of Icosavax.

Some vaccines,

such as those

for human

papillomavirus

and hepatitis B,

are produced

using viral

structural proteins

that naturally

Adam Simpson, CEO,

Icosavax (courtesy

Icosavax).

VLPs. "Those

have been some

of the most

effective vaccines

ever commercialized," Simpson adds.

Unfortunately, few naturally

self-assembling VLPs have been identified, and they won't necessarily accommodate any antigen you want to use to make a vaccine. But until now, there hasn't been a way to manufacture synthetic VLPs, with complex antigens attached, at scale.

Using a fully synthetic scaffold, King and his colleagues at IPD have been able to build different VLP configurations that contain various numbers of DS-Cav1 antigen molecules. The best configuration turned out to be an icosahedral structure, analogous to a soccer ball, with two different subunits, one of which has the antigen attached. The genius of their design is that the two subunits can be manufactured separately using conventional recombinant protein technology. When the subunits are mixed together, they self-assemble into the soccer-ball VLP with 20 copies of the antigens arrayed on the surface.

"You can stir them together and end up with this complicated macromolecular structure that wouldn't be manufacturable otherwise," Simpson says. The nanoparticles displaying the DS-Cav1 antigen induced an impressive antibody response both in mice and in primates, around tenfold higher than the antigen alone. In the future, the self-assembling VLP could be reconfigured to display other viral antigens in hopes of making vaccines for other formerly intractable diseases. Recently, King and his colleagues used the same approach to create a two-component self-assembling nanoparticle displaying stabilized versions of the HIV envelope protein. The particles stimulated a strong immune response in rabbits.

Whether the vaccine will be practical for use in vulnerable populations in the developing world, where RSV hits young

children hardest, will depend greatly on production costs. Jianrong Li of the Ohio State University suggests that live attenuated virus could be grown much more cheaply. "A virus is easy to grow, once you have an approved cell line," he ventures. Although the live attenuated virus vaccine isn't a viable product yet, if work by $\mathrm{Li}$ and others is successful, it may create competition for the synthetic vaccine. At that point, the cost of manufacturing and purifying the synthetic nanoparticle could become an issue.

But using synthetic self-assembling nanoparticles could afford vaccine designers much greater control over their final product, says Darrell Irvine, who works on immunological nanoparticles at the Massachusetts Institute of Technology. "Something that I think is exciting about their platform technology is the potential to explore variables, such as the particle size and the density of antigen that you want on the surface of the particle," he says. "When you're using natural existing viral platforms, you're stuck with whatever size and valency it normally has, whereas the Icosavax folks might be able to design particles that have optimal arrangements of antigen and particle sizes."

To develop synthetic nanoparticle vaccines, Icosavax raised $\$ 51$ million in series A funding in 2019 from an investor group that included Qiming Venture Partners USA, joined by Adams Street Partners, Sanofi Ventures and NanoDimension. "Incredible technology is necessary, but not sufficient," to attract investors, Simpson notes. A defense against RSV is an important unmet medical need for older adults, he points out, and combining the self-assembly technology with the RSV antigen made the perfect initial application. "We had a really great technology story, and we had a really great team of people," with a strong background in vaccine development and manufacturing, he says. Tadataka (Tachi) Yamada, former chief medical and scientific officer of Takeda Pharmaceuticals, chairs the board of directors.

"We think really carefully about when it's the right time to spin out," King says, because they want to ensure the venture has the best chance of success. "In this particular case, we had just gotten some data back where we put the RSV vaccine into primates," he recalls. "It recapitulated the extra-potent response we had seen in mice. That's when we said, ok, this thing is ready to go."

Icosavax is scaling up their RSV vaccine, aiming to start trials in 2021.

CS

Platelet BioGenesis: better than blood?

Platelets are perennially in short supply. Available only from human donations, they

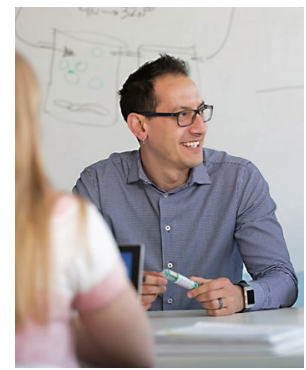

Jonathan Thon, cofounder and CSO, Platelet BioGenesis. have a short shelf life and are prone to contamination. Stem cell company Platelet BioGenesis aims to make platelets from iPSCs to boost supply. The market for thrombocytopenia treatment, including cancer care as well as trauma and other critical needs, is vast. Then graduate

student Jonathan Thon, working in the late 2000s to address supply problems in the laboratory of Dana Devine, the chief medical officer for Canadian Blood Services, realized there were limits to improving donor-derived platelets. "The challenge we have with platelet transfusions wasn't going to be solved by extending the lifespan of a platelet by a day or a couple of days," Thon says. "We fundamentally need to ... disconnect the product from the source material [human blood]. And ideally manufacture platelets ourselves."

Thon began attacking the problem as a postdoc in the Harvard lab of Joe Italiano, an expert in megakaryocyte and platelet biology. Megakaryocytes, which are resident in the bone marrow, grow to an enormous size and then release fragments in a highly orchestrated fashion. These cytoplasmic fragments become circulating platelets. In Italiano's lab and then his own lab, also at Harvard, Thon developed a platelet bioreactor to mimic this process. iPSCs provide the raw material. A ready source of well-characterized megakaryocytes, iPSCs can be maintained free from bacterial and viral contamination. In 2014, Thon, together with Italiano, microfluidics expert Linas Mazutis and venture capital investor Sven Karlsson, founded Platelet BioGenesis to make platelets from iPSCs. All are first-time founders.

For its first five years, until its \$26 million series A1 last September, Platelet BioGenesis was modestly funded. "This company wasn’t founded off of $\$ 50$ million from a major VC firm," says Thon. "This company was founded out of pocket, with the first couple thousands of dollars coming from myself and the cofounders." Largely sustained by angel investors and government grants, Platelet BioGenesis used its early years to redesign its bioreactor for industrial-scale manufacturing. Thon left his Harvard appointment in 2017 to become the company's CEO and CSO.

Platelets are a good stem cell tissue engineering test case. They're enucleate 
and terminally differentiated, so there's almost no cancer risk, and they can be safely irradiated to remove any contaminating nucleated cells. Platelets are also short-lived and minimally immunogenic, so allogeneic platelets are generally safe. "And the raw product itself is of critical therapeutic need," says Thon.

Platelet BioGenesis is building a whole platelet supply chain, from stem cell to final product. To begin with, "we have a GMP-compliant iPSC line that's been expanded and banked," Thon says. For differentiation into megakaryocytes, the company converted a lab-scale benchtop cell culture system into large-volume good manufacturing practice (GMP)-compliant bioreactors. Megakaryocytes will be frozen, then thawed on demand to make platelets in the company's platelet bioreactors.

Thon and Mazutis designed these platelet bioreactors to mimic the architecture of the bone marrow (although they do not recapitulate all marrow elements). Megakaryocytes are fed into a marrow-like space, from which they project long branching processes through a fenestrated series of gaps into an adjacent microfluidic channel modeled after blood vessels. There the projections are exposed to shear stress, which causes megakaryocytes to shed platelets.

All previous efforts to generate platelets outside the body for therapy have fallen short because megakaryocytes in culture make very few platelets. For therapy, massive numbers are needed; one unit of platelets, the standard dose, includes at least 300 billion platelets. "The Platelet Biogenesis approach has great potential for the treatment of low platelet counts," says Andrew Leavitt, medical director of the adult bone and marrow laboratory at the University of California, San Francisco. "I certainly hope they can achieve their goals, but at the end of the day, it's a numbers game, and that's a daunting task," he says.

A single natural mature megakaryocyte in vivo is capable of producing 1,000 or more platelets. In a March seminar, Italiano showed that his iPSC-derived megakaryocytes top out at 20 platelets each. Although that's an order of magnitude better than megakaryocytes in static cell culture, it falls two orders of magnitude short of natural megakaryocytes.

Platelet BioGenesis compensates by massively multiplexing its platelet bioreactors. The original bioreactor had a single microfluidic channel. This was multiplexed to sixteen channels, enabling production of 14 million platelets, according to Italiano's presented data. The company later stacked eight of these into 128-channel devices called 'blades'. The company is developing a 40-blade device, writes Thon in an e-mail. Simple math brings the platelet total to 4.5 billion platelets. Running 60 of these in parallel (or fewer devices running sequentially over several days or weeks) could in theory generate the 300 billion platelets required for a platelet unit. (Platelet BioGenesis declined to provide its own numbers.) "The company is focused on producing a platelet unit for first-in-human clinical studies," writes Thon, who says trials could begin "soon." (He declined to provide details.)

Platelet BioGenesis must first demonstrate to the US Food and Drug Administration (FDA) that its stem-cell-derived platelets look and perform like normal platelets. "Numbers are one thing, but the numbers must come with desired functionality," says Leavitt. "Both quality and quantity will be very important." In his March talk, Italiano reported that his bioreactor-generated platelets display platelet-specific markers, as well as the characteristic microtubule coils. They're also packed with the secretory granules typical of platelets.

Function is even more critical. These platelet-like cells must adhere to the damaged blood vessel wall, spread, aggregate, and form a plug that prevents bleeding. Platelet BioGenesis's iPSC-derived platelets show signs of working in two different mouse models. In one, a humanized mouse model of laser-induced vascular injury that allows human but not mouse platelets to function, "we observed primary hemostasis and a grade 1 adhesion event" with human iPSC-derived platelets, notes Italiano, whereas mouse platelets (the negative control) didn't work. In a second and more conventional mouse model, Italiano showed images of dye-labeled iPSC-derived platelets remodeling damaged tissue along with mouse platelets.

Platelet BioGenesis's main competitor (besides donor platelets) is Megakaryon, spun off in 2011 from Kyoto University in Japan. Megakaryon scientific founder Koji Eto invented a platelet production bioreactor similar in concept to the one Platelet BioGenesis is using, but that uses turbulence to boost platelet production. Megakaryon has developed immortalized cell lines to provide the megakaryocyte starting material.

Assuming Platelet BioGenesis solves the numbers problem and its bioreactor measures up to Megakaryon's, its platelets must also compete with donor platelets. This is a challenge, given the complexity and expense of the Platelet BioGenesis platelet supply chain, which includes the multiplexed devices and the necessary reagents, plus the culture media, growth factors and small molecules for differentiating stem cells into megakaryocytes. Although price point is a major market driver, Thon stresses other advantages to a stem cell product, mainly safety and availability. Donor platelets can be screened for viruses and bacteria, but unknown viruses will escape detection, and bacterial contamination is a constant risk because platelets must be stored at room temperature. And many poor countries have little access to platelets at all. "The best approach is actually to make platelets outside of the human body in large-scale bioreactors, that we could screen for bacteria and viruses, ensure are safe, and then make available [for] transfusions anywhere in the world," says Thon.

More long-term, Platelet BioGenesis is working on using platelets to deliver biological payloads for different therapeutic applications. That's because platelets have secretory granules containing angiogenic factors that in theory can be swapped out for therapeutics - for example, cancer drugs. Because of the many applications, "that's been talked about in the field for a long time," Leavitt says. But, he adds, "these are not small challenges."

\section{Elicio Therapeutics: Amph-lifying anticancer immunity}

The ideal destination for a cancer vaccine is the lymph node, where the $\mathrm{CD}^{+} \mathrm{T}$ cell activation necessary for a potent antitumor immune response takes place. When Massachusetts Institute of Technology biomedical engineer Darrell Irvine learned that cancer surgeons can highlight [sentinel] lymph nodes with small-molecule dyes to look for metastases, he saw an opportunity. These dyes bind tightly to albumin in interstitial fluid at the injection site, then traffic with the albumin to the lymph node. "We realized that this might be a strategy that one can intentionally build into therapeutics to send them to lymph nodes, and in particular that'd be useful for vaccines," says Irvine.

That albumin hitchhiking strategy led to the technology platform of Irvine's company, Elicio Therapeutics. Albumin has multiple lipid-binding pockets, so Irvine's group designed molecules comprising a diacyl-lipid tail linked to a payload - a tumor antigen for creating a vaccine or a ligand to amplify a chimeric antigen receptor (CAR)-T response - through a polyethylene glycol spacer. These amphiphilic 'amph-vaccines' are both hydrophilic (the spacer) and lipophilic (the tail).

Why an amphiphile? It must be hydrophilic to ensure solubility for transport to the lymph node. And it must be lipophilic so that, once at the destination, the lipid 
tails (with the cargo molecule) hop off the albumin, since that interaction is relatively weak, and then insert into the membranes of cells there - antigen-presenting cells (APCs) like macrophages and dendritic cells. In the vaccine application, APCs take up the antigen cargo, process it and present antigen peptides on MHC molecules for $\mathrm{T}$ cells to recognize and to become activated. The membrane insertion aspect is critical. "What that does is essentially help depot the [antigen/adjuvant payload] in the lymph node so it doesn't clear away, and helps it be captured and accumulate there," says Irvine. "If you give a traditional peptide immunization, the antigen is presented for a few days and then it's gone. But with the amphiphile, we see that antigen is still being presented for about 14 days. And that is probably the biggest factor that changes dramatically how strong the T cell response is."

In Elicio's adoptive cell therapy application, a CAR-T ligand, such as an epidermal growth factor receptor vIII mutant epitope, is the cargo. Elicio intends to apply this approach mainly against solid tumors, where CAR-T cells to date have worked poorly. The amphiphilic molecule, as in the vaccines, binds to albumin and traffics to the lymph node. Once there, the CAR-T ligand decorates APC membranes. CAR-T cells, injected separately, arrive in lymph nodes and see those ligands, along with "all of the other costimulatory receptors and cytokines being produced by that dendritic cell that would normally be a part of T cell priming," says Irvine. The CAR-Ts then proliferate. "You have more CAR-T cells, which is good, but also on a cell-by-cell basis they are more functional" because of the co-stimulation and cytokines, says Irvine. That, he adds, makes the CAR-Ts better able to deal with the immunosuppressive tumor microenvironment once they travel from the lymph node to the tumor.

Elicio began in 2011 as Vedantra Pharmaceuticals to commercialize a different lymph-node-targeting technology from Irvine's lab. The largest and founding shareholder is Clal Biotechnology Industries, which manages the biotech investments of British-American businessman Len Blavatnik. About three years ago, the company shifted completely to the amphiphile technology, and later changed its name to Elicio. A $\$ 6$ million series A round was complete by March, 2019, followed six months later by a $\$ 40$ million series B round.

Elicio plans to be in the clinic with a cancer 'amph'-vaccine sometime this year. The lead molecule employs a mutant KRAS antigen payload for pancreatic and colorectal cancers. It will be followed by

a head-and-neck cancer vaccine using human papilloma virus (HPV) antigens. (Both incorporate a CpG oligonucleotide adjuvant.) Meanwhile, the CAR-T amplifier is quickly moving forward. "We're actively talking to a number of potential academic and commercial partners," says Irvine. "I'm really hopeful that there's a short pathway to testing that in people in a year or two."

Elicio's amph-vaccine shows promise, says Bret Ulery, director of the Biomodulatory Materials Engineering Laboratory at the University of Missouri. "They've been able to show some really exciting data of the types of immune responses that they can generate," he says. However, competing approaches to the problem of vaccine lymph node trafficking have their own advantages. Particle vaccines,



Darrell Irvine, cofounder and scientific advisory board chairman, Elicio Therapeutics. which aggregate antigens within a polymeric or metallic particle, or in a liposome or other lipid complex, will also traffic to lymph nodes if they're stable and the right size. BioNTech, for example, is testing a RNA-lipoplex vaccine in clinical trials for malignant melanoma. Particles can individually be more efficient: each

time a cell internalizes a nanoparticle, says Ulery, hundreds or thousands of peptide antigens are released, whereas with the Elicio amphiphilic molecule, which delivers a single peptide or protein, "the cell's going to have to take up a lot of those to hit a critical mass for presentation," he says. With particle vaccines, "it's going to take less of those interactions to achieve whatever therapeutic dose you need."

Irvine says getting a sufficient dose of his amph-vaccine is not an issue. "These amphiphiles so efficiently enter the lymph node, they're just everywhere," he says. "So it doesn't matter that it's just one molecule. We're putting thousands of molecules on each APC." Irvine, who has worked a lot with particulate vaccines, says he's never been able to make one that competes with the amphiphile technology. That, he says, may be because particles tend to be captured by macrophages or dendritic cells for degradation, whereas amphiphiles persist on the APC cell surface, resist degradation and are more efficiently processed for MHC presentation.

Ulery acknowledges the drawbacks of particles, but notes that only clinical testing can determine whether amphiphiles are better. "The best way to approach it, I don't think that's been solved yet," he says. A larger issue, with all cancer vaccines (not just amph-vaccines), is immunosuppression at the tumor. Elicio's amphiphile "just may not be effective," says Ulery. "You may still mount an immune response, it maybe helps a little bit, but then either the cancer cells are under selective pressure and transitioning so they're no longer expressing that [antigen]. Or if it's a solid tumor, it's a complex environment to try and get active cytotoxic T cells in."

Irvine knows the obstacles, but is encouraged that the amph-vaccine, unlike virtually all other cancer vaccines, actually works in rigorous preclinical animal models. In work published in 2014, his group tested the vaccine in two different mouse tumor models: TC-1 tumors, expressing the HPV oncoprotein E7, and the B16F10 melanoma model. In both models, amphiphile vaccines outperformed soluble vaccines that lacked the amphiphilic backbone.

Elicio's CAR-T amplifier faces fewer obstacles than the vaccines because potent antitumor T cells are already present, as they've been injected in the circulation. In preclinical models of the CAR-T amplifier, Irvine has observed antigen spreading at the tumor, whereby the amplified CAR-T cells promote an endogenous antitumor immune response. Irvine speculates that the manner in which these CAR-T cells kill, or the factors they secrete, promote such antigen spreading, which is a key to all immunotherapy success in cancer.

There are two toxicity concerns. One is whether the CAR-T cells will wipe out the APCs displaying the CAR-T ligand in the lymph node, and the other is whether the extra CAR-T stimulation will cause autoimmune side effects. Irvine didn't see APC killing in his models. "Perhaps dendritic cells in the lymph node have some means to protect themselves," he says. "We're still working to understand that better." Nor was there triggering of autoimmunity. But Elicio also faces potential competition from a BioNTech particulate CAR-T booster, among many other approaches now in development to improve CAR-T cell therapy.

Raising money for Elicio, until recently, was difficult, says CEO Bob Connelly, because of the stigma attached to the cancer vaccine space after decades of failures. Talking to potential investors, "I can't tell you how many times I've heard, 'Oh, we've got scars here from cancer vaccines,' he says. Elicio, after the KRAS and HPV projects, is not developing more vaccines, says Connelly. Instead, it will advance cell therapy applications of the amphiphile 
technology, beginning with the CAR-T amplifier. Investors in the cell therapy space "are much more willing to get involved at an earlier stage," Connelly says. "It doesn't carry the baggage that cancer vaccines have."

First, the company must show that its amph-vaccines or its CAR-T amplifier, or both, work in people. If they do, other cell therapy applications will follow. Lymph node targeting, says Connelly, is applicable "in any cell therapy, any immunotherapy, that really relies on an immune response."

\section{Twentyeight-seven: drugging RNA-protein interactions}

Editing DNA and RNA might be all the rage, but if Kazumi Shiosaki, CEO of the newly emerged Twentyeight-seven,

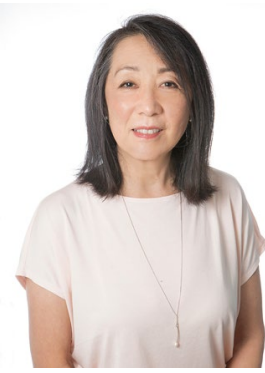

Kazumi Shiosaki, president and CEO, Twentyeight-seven. Credit: Kim Furnald

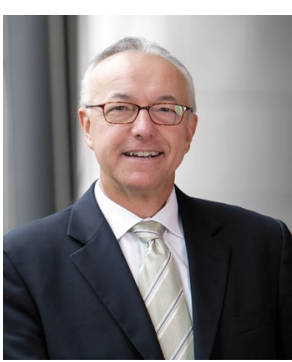

George Daley, dean of Harvard Medical School, cofounder, Twentyeight-seven (courtesy Harvard Medical School). is right, there's still a place for small molecules in targeting nucleic acids. Teaming up with four heavyweights from Harvard Medical School - George Daley, Richard Gregory, Piotr Sliz, and Frank Slack the company has come out of the gates with a sizeable A round of $\$ 82.8$ million to put this idea into practice.

The venture had been gestating for over a decade, beginning in 2006 in George Daley's lab. His student Srinivas Viswanathan obtained evidence that the let-7 family of microRNAs, which are tumor suppressors, is developmentally regulated. About

that time, Richard Gregory, an expert in the biochemistry of microRNA processing, was recruited to Harvard Medical School. With that capability, they found that a developmentally regulated protein, Lin28, blocked let-7 microRNA processing in embryonic stem cells. Because let-7 is a tumor suppressor, this meant that Lin28, as a negative regulator of a tumor suppressor, is itself an oncogene, Daley surmised.
The next question then would be how to attack the complex, which became tractable after Piotr Sliz and his colleagues solved in 2011 the cocrystal structure of Lin 28 with let-7. They started speculating on how to screen for molecules that interfere with the Lin28-let-7 interaction, which they reasoned would be candidates for anticancer therapies. Once they recruited Frank Slack, an RNA specialist, into the community, the team was complete.

As the four contemplated where they might get funding for this project, they realized that they might be able to get outside funding to establish a company. Daley, who had a long-standing relationship with MPM Capital, had in fact pitched this idea a decade ago. That brought in Shiosaki, a principal at MPM Capital, who also had a long-standing relationship with Daley, as they both were involved with launching Epizyme in 2007. Shiosaki, who came up through the ranks of medicinal chemistry, was excited at the prospect of addressing dysregulation of RNA with small molecules. "Most RNA is associated with protein; that's where George Daley's finding with Lin28, a protein that regulates the formation of the mature let-7, was seminal. It was that association of this protein and RNA which got us thinking," she says. "Could a small molecule that inhibits that association between Lin 28 and let7 allow for the precursor of let-7 to go on its merry way and be processed to the mature let-7?" she wondered. If so, such a molecule could turn out to have potent anticancer properties.

Although Shiosaki wouldn't provide any specifics, she says that they are finding molecules that work in the Lin28-let-7 pathway. In addition, she sees a potential to move beyond cancer into certain inflammatory or fibrotic disorders where there is dysregulation of the same components. And finally, they are on the hunt for other RNA-binding proteins that can be targeted with small molecules. "It's early, and we haven't disclosed them publicly yet; nonetheless, we have indeed identified other exciting RNA-binding proteins," Shiosaki says.

Vijay Sankaran at Harvard's Stem Cell Institute is working on exploiting Lin28's control over developmental processes in his specialty, hematology. He has found that Lin28 is involved in the switch to adult hemoglobin from fetal hemoglobin, a process that many researchers would like to control to treat various hemoglobin disorders. He does have one caveat, however: as Lin28 has pleotropic properties, it might be a double-edged sword, and there may be some settings in which you won't want to use it. Nonetheless, he is very enthusiastic about being able to interfere with protein-RNA interactions in processes like translation, and has high hopes that it will bear fruit for his patients.

Shiosaki likes taking charge of companies when there's little more than air - putting together all the pieces that have to come together to get the seed or series A, get the first couple of hires, get the space, get the first assay up and running. And for Daley, there couldn't be a better partner. "I would do anything with Kazumi. She's a thoughtful, smart medicinal chemist trained in organic chemistry. She also has amazing entrepreneur chops," he says.

As dean at Harvard Medical School, Daley was frustrated at how difficult it is to develop a therapeutic within academia and is setting up programs to try to fill some of the gaps. "If this project hadn't been picked up as a newco seeded initially by a joint venture by MPM and Astellas, I believe it might have withered on the vine," he says.

\section{Shape Therapeutics and Korro Bio: RNA editing in the spotlight}

Two decades-old observations are now coming to the fore as RNA editing comes out of the shadow of DNA editing and takes a place at investors' tables. Back in 1995, Tod Woolf, Jennifer Chase and Dan Stinchcomb first demonstrated how to edit RNA with oligonucleotides. Not long after, Josh Rosenthal, while doing his graduate work at Stanford, discovered that cephalopods recode RNA in their neurons. At the time, it wasn't obvious that these discoveries could lead to a human therapeutic - it took months of work for Rosenthal to clone a single sequence - but with today's technology, it's apparently a no-brainer.

Last year, Beam Therapeutics and Locana emerged with plans for base editing of DNA and RNA with CRISPR-based systems. This year, two companies, Shape Therapeutics and Korro Bio, have turned to a different RNA-modifying enzyme, adenosine deaminase working on RNA (ADAR), one that is endogenous and ubiquitous in human cells. RNA editing with ADAR has several advantages over some DNA-editing systems; high among them is that a human enzyme would not create immunogenicity problems that bacterially derived, CRISPR-based systems using bacterial endonucleases might.

The enzyme at the center of all this activity, ADAR, converts adenosines to inosines, which are read as guanosine during translation, essentially converting an A to a G. Rosenthal discovered that cephalopods have a penchant for using this activity in their nervous systems when he was cloning ion channel genes from cDNAs 




Josh Rosenthal, cofounder, Korro Bio.

Credit: Tom Kleindinst

isolated from squid giant axons (the quintessential system for ion channel studies). He couldn't get a consistent sequence among different clones of the same message and, upon closer analysis, discovered G-to-A

variations at specific points in the sequence. "They recode like crazy," Rosenthal says. Later, after Rosenthal set up his own lab at the Institute of Neurobiology, part of the University of Puerto Rico Medical Sciences Campus, a student embarked on a project to correct a cystic fibrosis mutation, using ADAR that had a $\lambda$-phage protein linker attaching an antisense oligo to the catalytic (deaminase) domain. They targeted the oligo to a premature stop codon in a mutated cystic fibrosis transmembrane conductance regulator (CFTR) gene and road tested the construct in Xenopus oocytes, a convenient translation system. Despite the groundbreaking aspect of the work, it was largely ignored.

Rosenthal has continued to work on recoding RNAs in cephalopods using ADAR, with new generations of engineered enzyme. His approach of exogenously supplying the enzyme will enable him to edit in cells or tissues that express low levels of the endogenous enzyme. He also hopes to broaden the range of sites that can be targeted; the enzyme requires certain nucleotides surrounding the 'A' of interest. By tinkering with the enzyme, Rosenthal believes he can overcome some of these limitations.

Meanwhile, Rosenthal had moved his lab to Marine Biological Laboratory in Woods Hole, Massachusetts, now a unit of the University of Chicago. A year later, Rosenthal



Nessan Bermingham, cofounder and executive chairman, Korro Bio (courtesy Korro Bio). found himself presenting his work before an investor group at nearby Cambridge, as the powers to be at the university sought to exploit some of the research in their new asset. Seated at the table was Nessan Bermingham, former CEO of
Intellia Therapeutics, a CRISPR-based DNA editing company, and the rest, as they say, is history. Bermingham is now executive chairman of Korro Bio, which licensed Rosenthal's system. "CRSPR had been around long enough, things had gone forward, they saw limitations. It just made sense to them that RNA editing could be a very potent place for intervention," says Rosenthal.

Bermingham, only recently freed from his responsibilities as CEO of Intellia, wasn't planning on taking on a new company just yet but, as a member of Atlas Venture, became involved in seed funding of an RNA-editing project. Once he saw that several issues could be addressed - that specific 'A' residues could be targeted, not 10 bases plus or minus, and that ADAR could be delivered in vivo - he and Atlas, along with buy-in from NEA, were ready to launch a new company.

The Korro management team hasn't disclosed which indications it plans to pursue, but Bermingham is currently focused on oligonucleotides to simplify delivery and manufacturing. His ideal would be to co-opt the body's endogenous RNA-editing system, if it could be made efficient enough, to avoid making permanent DNA edits, the long-term consequences of which are unknown. "The prudent thing thinking about the safety of patients is going in transiently to verify the target is appropriate and then go in with a more permanent modification," he says. Bermingham says that they will initially focus on editing G-to-A mutations back to a $G$ in genetic disease, with a look to the future in targeting protective mutations or editing post-translational modification sites to modulate specific protein function.

Shape Therapeutics was formed by two researchers who had forged a friendship while in George Church's lab back in the early 2010s, Prashant Mali and Francois Vigneault. Mali, after working on CRISPR in the lab - leading to the first engineering of CRISPR systems for human genome engineering - was looking for a system that was easier to work with, one that was "more democratic in the sense of making it an easier tool," he says. He still works on and likes zinc fingers. But, in thinking about gene-therapy applications, he wanted to get away from prokaryotic systems with their inherent immunogenicity, and was interested in finding a system in humans that could do the same things. That brought him to the Stinchcomb paper, which provided the inspiration to use simple oligonucleotide hybrid molecules to edit



Prashant Mali, cofounder, Shape Therapeutics. Credit: Chitra Gopalakhrisnan
RNA. In a 2019

Nature Methods paper, Mali succeeded for the first time in editing RNA in vivo using ADAR activity.

Vigneault says of his friend and now cofounder, "Prashant did something totally unique, using a gene-encoded guide RNA to recruit endogenous ADAR, but in a way that you could do a therapeutic treatment. He is the only one who has shown in vivo a proof of concept." In the paper, Mali edited RNAs in two mouse models: Duchenne's muscular dystrophy, where he was able to restore expression of the gene for dystrophin by recoding a mutation that led to premature termination of translation; and ornithine transcarbamylase deficiency. This work, according to Vigneault, was the triggering event for launching the company.

Shape is working on three fronts. The first is the design of the RNA payload and how to optimize secondary structure to maximize recruitment of ADAR. The second is a delivery method, based on a novel adeno-associated virus (AAV)- 5 system, that triggers less immunity than other AAV species. Shape has generated a library of $10^{11}$ AAVs that will be tested in non-human primates to see where they end up. The third is manufacturing, where he hopes to improve on present platforms, in which only a small percentage of the product is active. The company also licensed an engineered tRNA that replaces a nonsense mutation, which provides an alternative approach to targeting particular mRNAs.

It's still early days for RNA-editing companies, but for the researchers in this field, the world around them has caught up. "We were this kind of weird offshoot; we didn't even have our own study sections at NIH," Rosenthal says. Now, at last year's Gordon Conference on RNA editing, he says there was a biotech presence for the first time.

\section{Michael Eisenstein ${ }^{1}$, Ken Garber²,} Caroline Seydel ${ }^{3}$ and Laura DeFrancesco ${ }^{4}$ ${ }^{1}$ Philadelphia, PA, USA. ${ }^{2}$ Ann Arbor, MI, USA. ${ }^{3}$ Los Angeles, CA, USA. ${ }^{4}$ Senior Editor, Nature Biotechnology.

Published online: 23 April 2020 https://doi.org/10.1038/s41587-020-0506-3 